\title{
Spontaneous Construction of Voronoi Diagram for Polygon
}

\author{
Ye Zhao \\ Department of Mathematics and Physics \\ Shijiazhuang Tie Dao University, Shijiazhuang \\ Hebei, China \\ E-mail: ye_box@163.com
}

\begin{abstract}
Voronoi diagram for polygon is difficult to construct because polygons have Irregular boundary consisting of segments. In traditional algorithm, when generators of polygons are complex, production process will be extremely complex because of the complex relationship between line segments. In this paper, we use spontaneous construction of Voronoi diagrams. The algorithm can get over all kinds of shortcomings that we have just mentioned. So it is more useful and effective than the traditional algorithm. The results show that the algorithm is both simple and useful, and it is of high potential value in practice.
\end{abstract} words)

Keywords- Voronoi diagram, Spontaneous, Polygon (key

\section{INTRODUCTION}

We have already noted that the concept of the Voronoi diagram is used extensively in a variety of disciplines and has independent roots in many of them. Voronoi diagram was appeared in meteorology, biology discipline and so on [1-3]. Now, people extend generator from a point to a line, a circle, or a polygon. More and more people pay attention to the algorithm that can construct Voronoi diagram fast and effectively. The traditional algorithms [4-6] have many Shortcomings. While spontaneous construction of Voronoi diagrams can get over many kinds of them.

\section{DEFINITIONS}

Wherever etc.

$$
\begin{aligned}
& \text { Let } P=\left\{p_{1}, p_{2}, \cdots, p_{n}\right\} \subset R^{2} \text {, where } \\
& 2<n<+\infty \text { and } x_{i} \neq x_{j} \text { for } i \neq j, i, j \in I_{n} \text {. We }
\end{aligned}
$$
call the region given by

$$
V\left(p_{i}\right)=\left\{x \mid\left\|x-x_{i}\right\| \leq\left\|x-x_{j}\right\| \text { for } j \neq i, j \in I_{n}\right\}
$$

the planar ordinary Voronoi polygon associated with , and the set given by

$$
\mathrm{V}=\left\{V\left(p_{1}\right), V\left(p_{2}\right), \cdots, V\left(p_{n}\right)\right\} \text {. }
$$

The planar ordinary generated by $P$ (or the Voronoi diagram of $P$ ). We call $p_{i}$ of $V\left(p_{i}\right)$ the generator point or generator of the ith Voronoi, and the set the generator set of the Voronoi diagram $\mathrm{V}$ (in the literature, a generator point is sometimes referred to as a site) [7], as shown in Fig. 1.

\author{
Xin Liu \\ Department of Mathematics and Physics \\ Chengde Petroleum College \\ Hebei Chengde, China \\ e-mail:Liuxincd@163.com
}

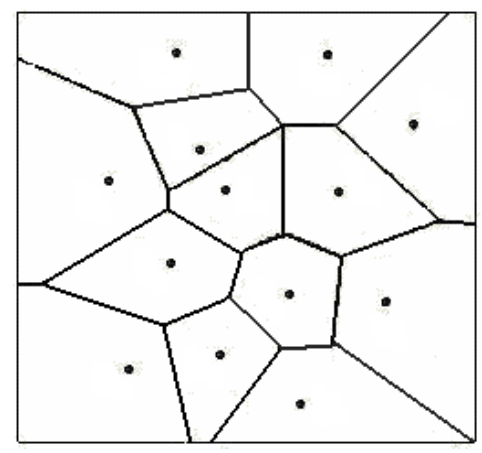

Figure 1. Degenerate Voronoi diagram

We assume that a generator $P_{i}$ is a polygon consisting of straight line segments. The shortest distance between $p$ and a straight line segment $P_{i}$ is defined following.

$$
\begin{aligned}
& d_{S}\left(p, P_{i}\right)= \\
& \left\{\begin{array}{l}
\left\|x-x_{i 1}\right\|_{i 2} \| \\
\left\|\left(x-x_{i 1}\right)-\frac{\left(x-x_{i 1}\right)^{T}\left(x_{i 2}-x_{i 1}\right)}{\left\|x_{i 2}-x_{i 1}\right\|}\left(x_{i 2}-x_{i 1}\right)\right\|
\end{array}\right. \\
& \text { if } p \in R_{i 1} \\
& \text { if } p \in R_{i 2} \\
& \text { if } p \in R_{i 3}=R^{2} \backslash\left[R_{i 1} \cup R_{i 2}\right]
\end{aligned}
$$

Where $x_{i 1}$ and $x_{i 2}$ are the end point of $P_{i}$, and $R_{i 1}=\left\{x \mid\left(x_{i 2}-x_{i 1}\right)^{T}\left(x-x_{i 1}\right)<\mathbf{0}\right\}$ $R_{i 2}=\left\{x \mid\left(x_{i 1}-x_{i 2}\right)^{T}\left(x-x_{i 2}\right)<\mathbf{0}\right\}{ }^{[8]}$

(A line Voronoi diagram generated by set consist of two polygons is shown in Fig. 2 


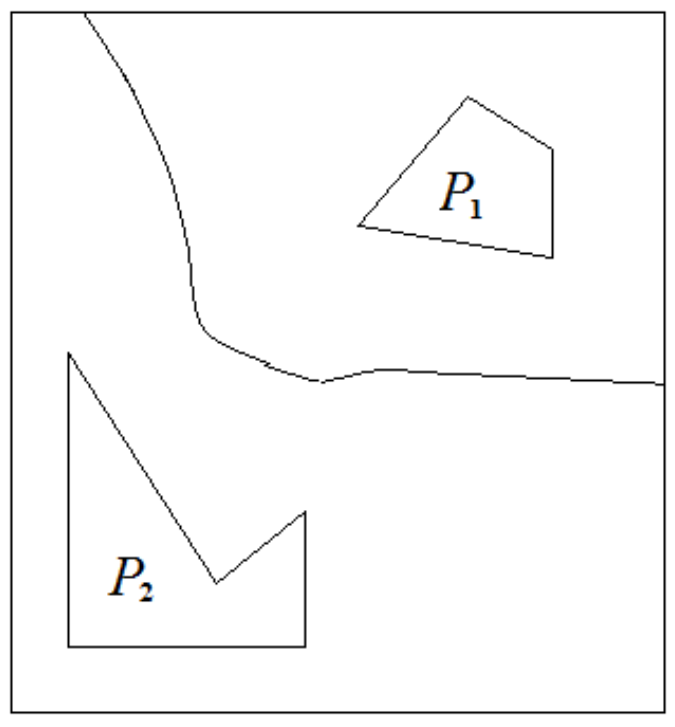

Figure 2. A Voronoi diagram of two polygons

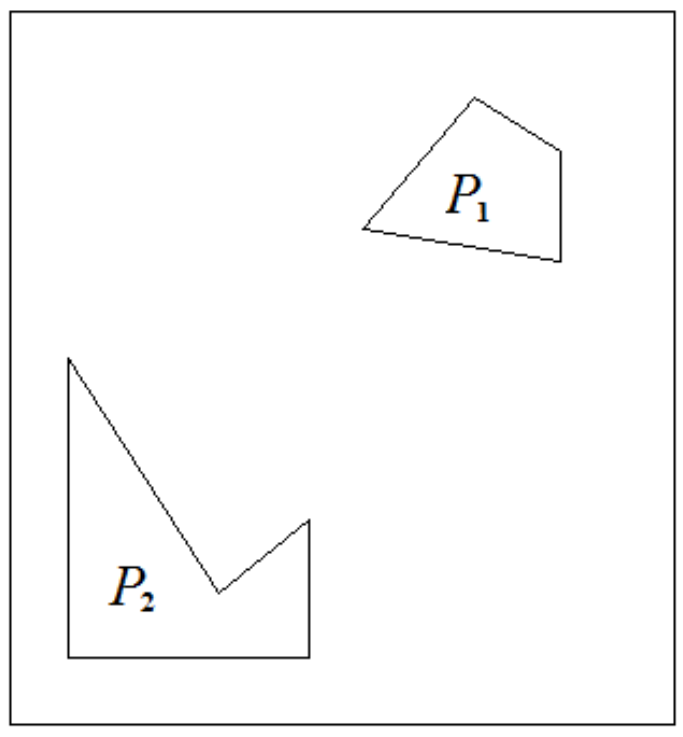

Figure 3-1. Two Generators of Polygons

\section{CONSTRUCTION OF SPONTANEOUS VORONOI DIAGRAMS FOR FIGURES}

In the actual situation, we can frequently encounter that generator of polygon changes. Production process will be extremely complex because of the change of regions neighboring with those changed generator this time[9]. We can get over many kinds of them by using spontaneous construction of Voronoi diagrams.Here we construct Voronoi diagram with spontaneous algorithm. Firstly, we assign different colours for different generators. Then choose some points on those generators. Finally, use spontaneous algorithm constructing Voronoi diagram. The procedure end when all points on screen are marked colour. This time, we get the Voronoi diagram [10].

Voronoi diagrams for polygons were widely used on geographic information system and urban planning. Figure

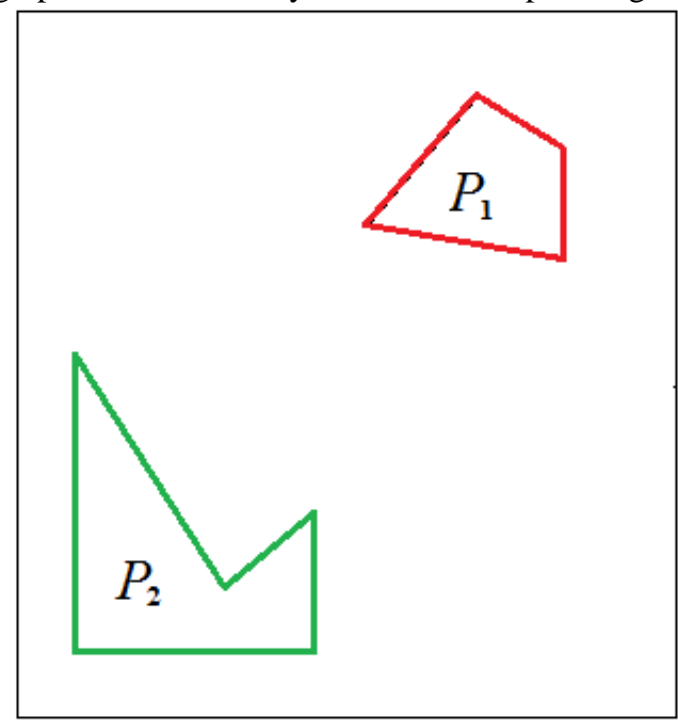

Figure 3-2. Assign Different Colour for Different Generator

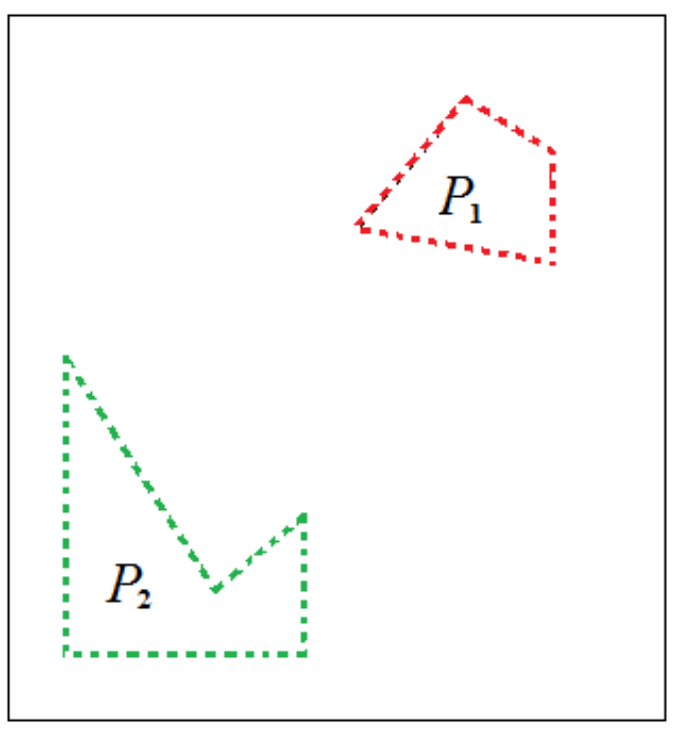

Figure 3-3. Choose Some Points on the Generators

3 show us the generation of process. We take two generators of polygons as the example (Figure 3-1). Construct Voronoi diagram using spontaneous algorithm. Firstly, we assign different colour for different generator. Here polygon P1 was assigned to be red and polygon P2 green (Figure 3-2). Then choose some points on the generators to represent generator themselves (Figure 3-3). Next draw circles with taking generator points as the centre and distance as radius (Figure 3-4). The program ends when screen of all pixel are assigned 
colour (Figure 3-5). At last, we assign generators black and assign other pixels white, and Voronoi diagram for polygons is got (Figure 3-6).

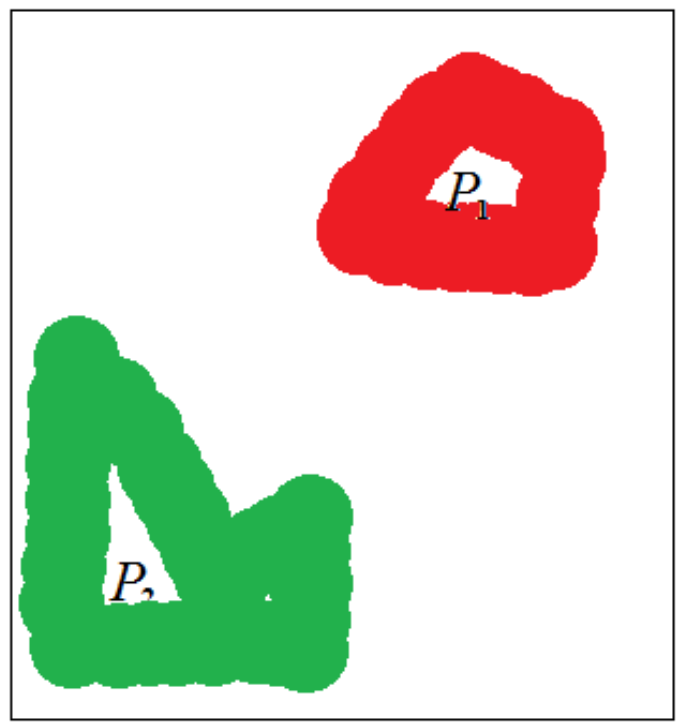

Figure 3-4. Draw Circles with Taking Generator Points as the Centre and Distance as Radius

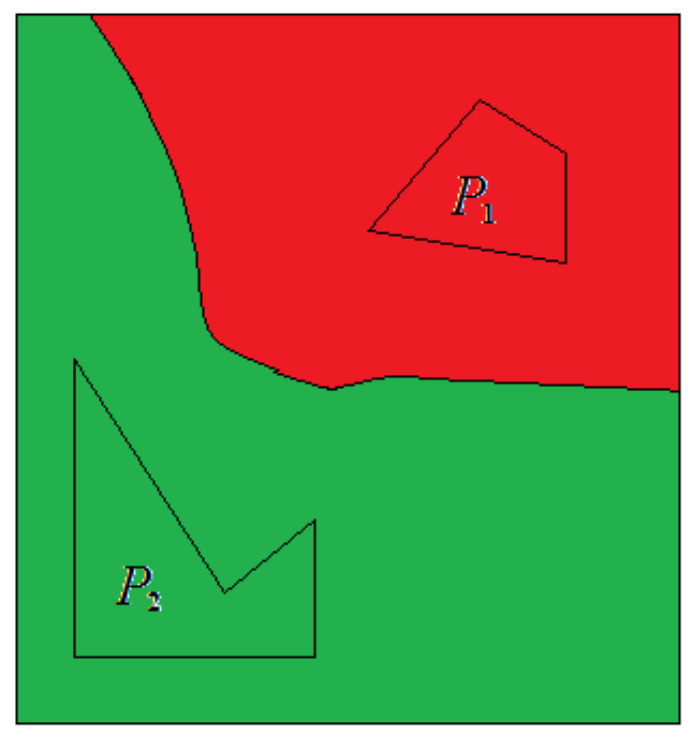

Figure 3-5. Screen of all Pixel are Assigned Colour

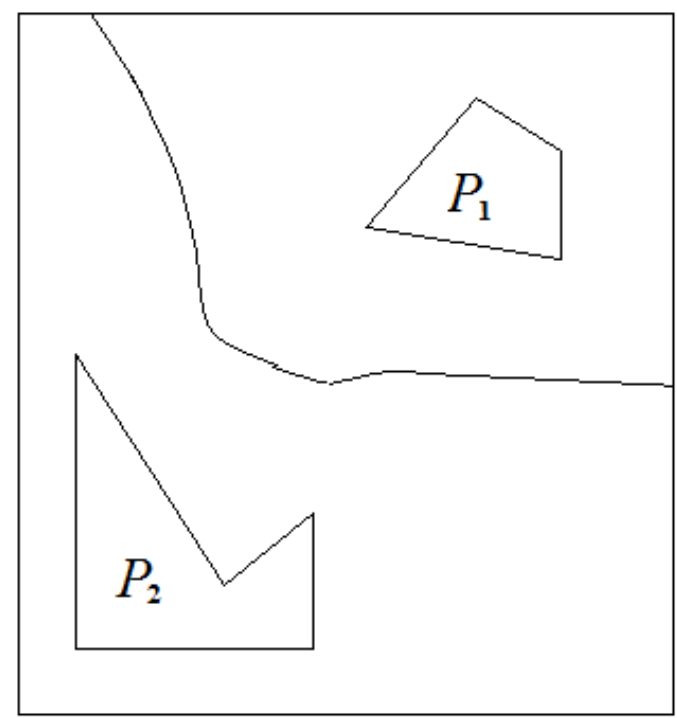

Figure 3-6. Voronoi diagrams for polygons

\section{CONCLUSIONS}

The construction of Voronoi diagrams for polygon by spontaneous algorithm can get over many kinds of shortcomings, because we need only to consider the generator changed. So it is more useful and effective than the traditional algorithm. The results show that the algorithm is both simple and useful, and it is of high potential value in practice.

\section{ACKNOWLEDGMENT}

The Youth Foundation of Hebei Educational Committee, grant (QN20131159)

The Foundation of Hebei Educational Committee, grant (Z2013113)

\section{REFERENCES}

[1] Kazuhiro Yamada and Kokichi Sugihara. "Approximation of Voronoi diagrams for figures and its improvement”, Department of Mathematical Engineering and Information Physics, Graduate School of Engineering, University of Yokyo,1996

[2] Takafumi Watanabe and Sadayuki Murashima."A method to construct a Voronoi diagram on 2-D digitized space (1) computing time” . Faculty of Engineering, Kagoshima

[3] Voronoi,G.Nouvelles. “ applications des parameters continues a la theorie des formes quadratiques”, Deuxieme memoire:Recherches sur les parplleloedres primitives. Math, 1908, (134):198-287

[4] Edetsbrunner H. "Smooth surfaces for multiscale shaperepresentation", Proceedings of the 15th Conference on Foundations of Software Technology and Theoretical Computer Science, Bangalore, 1995. 391-412

[5] Yang Cheng-Lei, Wang Jia-Ye, Meng Xiang-Xu,. "Upper Bounds on the Size of Inner Voronoi Diagrams of Multiply Connected Polygons”, Journal of Software, 2006.7,17(7):1527-1534

[6]. Jun Chen, Renliang Zhao, Zhilin Li.: Voronoi-based k-order neighbour relations for spatial analysis. J. ISPRS Journal of Photogrammetry and Remote Sensing. 59, 60—72 (2004) 
[7]. Ickjai Lee, Kyungmi Lee.: A generic triangle-based data structure of the complete set of higher order Voronoi diagrams for emergency management. Computers, Environment and Urban Systems. 33, 90-99 (2009)

[8]. S. Cabello, M. Fort, J.A. Sellarès.: Higher-order Voronoi diagrams on triangulated surfaces. J. Information Processing Letters. 109, 440-445 (2009)

[9]. Yugong Wu, Weibin Zhou, Bu Wang, Fan Yang.: Modeling and characterization of two-phase composites by Voronoi diagram in the Laguerre geometry based on random close packing of spheres. Computational Materials Science. 47, 951--96, 2010

[10]. Ye Zhao, Shujuan Liu, Youhui Zhang. Spatial Density Voronoi Diagram and Construction. Journal of Computers. Vol 7, (2012), 2007-2014, Aug 2012.

[11] Wu Ran,Ye Zhao. Discrete Construction of Network Voronoi Diagram. ICMCI'2010. : 630-632. 